\title{
Trastuzumab With S-1 Plus Cisplatin in HER2-positive Advanced Gastric Cancer Without Measurable Lesions: OGSG 1202
}

\author{
SHUNJI ENDO ${ }^{1,2}$, YUKINORI KUROKAWA ${ }^{3}$, MAKIO GAMOH ${ }^{4}$, YUTAKA KIMURA ${ }^{5}$, \\ JIN MATSUYAMA ${ }^{2}$, HIROKAZU TANIGUCHI ${ }^{6}$, ATSUSHI TAKENO ${ }^{7}$, RYOHEI KAWABATA $^{8}$, \\ JUNJI KAWADA ${ }^{9}$, TORU MASUZAWA ${ }^{10}$, KAZUYOSHI YAMAMOTO ${ }^{11}$, \\ KOUJI KOBAYASHI ${ }^{12}$, DAISUKE SAKAI ${ }^{13}$, TOSHIO SHIMOKAWA ${ }^{14}$ and TAROH SATOH ${ }^{13}$ \\ ${ }^{1}$ Department of Gastroenterological Surgery, Higashiosaka City Medical Center, Osaka, Japan; \\ ${ }^{2}$ Department of Surgery, Yao Municipal Hospital, Osaka, Japan; \\ ${ }^{3}$ Department of Gastroenterological Surgery, Osaka University Graduate School of Medicine, Osaka, Japan; \\ ${ }^{4}$ Department of Medical Oncology, Osaki Citizen Hospital, Miyagi, Japan; \\ ${ }^{5}$ Department of Gastroenterological Surgery, Sakai City Medical Center, Osaka, Japan; \\ ${ }^{6}$ Department of Surgery, Minoh City Hospital, Osaka, Japan; \\ ${ }^{7}$ Department of Gastroenterological Surgery, Kansai Rosai Hospital, Hyogo, Japan; \\ ${ }^{8}$ Department of Surgery, Osaka Rosai Hospital, Osaka, Japan; \\ ${ }^{9}$ Department of Surgery, Kaizuka City Hospital, Osaka, Japan; \\ ${ }^{10}$ Department of Surgery, Osaka Police Hospital, Osaka, Japan; \\ ${ }^{11}$ Department of Surgery, National Hospital Organization Osaka National Hospital, Osaka, Japan; \\ ${ }^{12}$ Department of Internal Medicine, Showa University Northern Yokohama Hospital, Kanagawa, Japan; \\ ${ }^{13}$ Department of Frontier Science for Cancer and Chemotherapy, \\ Osaka University Graduate School of Medicine, Osaka, Japan; \\ ${ }^{14}$ Clinical Study Support Center, Wakayama Medical University, Wakayama, Japan
}

\begin{abstract}
Background/Aim: Trastuzumab with S-1 plus cisplatin was proved to be effective for human epidermal growth factor receptor type 2 (HER2)-positive advanced gastric cancer with measurable lesions. However, the efficacy and safety of this regimen in the absence of measurable lesions are unknown. Patients and Methods: Patients with HER2-positive gastric cancer without measurable lesions received cisplatin plus trastuzumab intravenously on day 1 and oral S-1 on days 1-14 of a 21day cycle. The primary end-point was overall survival, and 40 patients were planned to be enrolled. Results: Fifteen patients were enrolled. The median overall survival was 14.4 months. The 1- and 3-year overall survival rates were $66.7 \%$
\end{abstract}

Correspondence to: Yukinori Kurokawa, Department of Gastroenterological Surgery, Osaka University Graduate School of Medicine, 2-2, Yamadaoka, Suita, Osaka, Japan. Tel: +81 668793251, Fax: +81 668793259, e-mail: ykurokawa@gesurg.med.osaka-u.ac.jp

Key Words: Gastric cancer, human epidermal growth factor receptor type 2, trastuzumab, S-1, cisplatin. and $26.7 \%$, respectively. Major grade 3-4 adverse events included neutropenia (47\%), anemia (40\%), diarrhea (20\%), nausea (20\%), and anorexia (20\%). Conclusion: Trastuzumab with $S-1$ plus cisplatin might be effective and tolerable for HER2-positive advanced gastric cancer without measurable lesions.

Gastric cancer is estimated to be the third most common cause of cancer-related death worldwide in 2018 (1). Although the number of patients who have died of gastric cancer has gradually decreased over the past decade in Japan (2), the prognoses of patients with unresectable advanced gastric cancer (AGC) are still poor.

For human epidermal growth factor receptor type 2 (HER2)-positive AGC, the ToGA trial (3) demonstrated a survival benefit from the addition of trastuzumab to chemotherapy consisting of capecitabine or fluorouracil plus cisplatin, while S-1 plus cisplatin (SP) had been considered as standard chemotherapy regimen for AGC in Japan since the SPIRITS trial (4). As trastuzumab in combination with SP had not been evaluated in patients with HER2-positive AGC, the HERBIS-1 phase II trial (5) was conducted to evaluate the 
efficacy and safety of SP plus trastuzumab in HER2-positive AGC with measurable metastatic lesions according to the Response Evaluation Criteria in Solid Tumors (RECIST) version 1.1 (6). It showed that $\mathrm{SP}$ plus trastuzumab had a promising antitumor activity and manageable toxic effects.

However, some AGC patients do not have measurable lesions according to the RECIST 1.1 and such a population was excluded in the HERBIS-1 trial. In the subgroup analysis of the ToGA trial, trastuzumab plus chemotherapy failed to provide a survival benefit for patients without measurable lesions compared with chemotherapy alone. Although other phase II trials were carried out to examine the efficacy of trastuzumab in combination with chemotherapy (7-16), no data were available for patients with HER2-positive AGC without measurable lesions. Thus, the efficacy of the addition of trastuzumab to chemotherapy for AGC without measurable lesions is still unknown.

According to the subgroup analyses of the SPIRITS trial, the effects of SP on overall survival (OS) were greater in patients without target tumors than in those with target tumors (4). This suggested SP to be effective for OS in patients without measurable lesions. Therefore, we conducted a single-arm, multicenter phase II trial, HER2 Based strategy In Stomach cancer (HERBIS)-1B, to evaluate the efficacy and safety of SP plus trastuzumab for HER2positive AGC without measurable metastatic lesions.

\section{Patients and Methods}

Patients. We enrolled patients with histologically confirmed unresectable or recurrent gastric adenocarcinoma including adenocarcinoma of the gastro-esophageal junction. The criteria for eligible patients included those with no measurable legion as defined by RECIST 1.1 within 21 days before enrollment, patients with HER2-positive cancer confirmed by immunohistochemistry (IHC) and/or fluorescence in situ hybridization (FISH) (IHC 3+ or IHC $2+$ and FISH positive), age 20-75, performance status (PS) 0 or 1 (ECOG scale), no prior chemotherapy or radiotherapy for gastric cancer (recurrence more than six months after adjuvant chemotherapy was accepted), no massive ascites or pleural effusion retention, patients without brain metastasis, adequate baseline organ and marrow function with leukocytes $\geq 3,500 / \mathrm{mm}^{3} \leq 12,000 / \mathrm{mm}^{3}$, absolute neutrophil count $\geq 2,000 / \mathrm{mm}^{3}$, platelets $\geq 100,000 / \mathrm{mm}^{3}$, hemoglobin $\geq 9.0 \mathrm{~g} / \mathrm{dl}$, AST $(\mathrm{GOT}) /$ ALT $(\mathrm{GPT})<100 \mathrm{IU} / 1$, total bilirubin $<1.5 \mathrm{mg} / \mathrm{dl}$, serum creatinine $\geq 1.2 \mathrm{mg} / \mathrm{dl}$, and creatinine clearance $\geq 60 \mathrm{ml} / \mathrm{min}$, patients with a left ventricular ejection fraction of at least $50 \%$ on Multi Gated Acquisition Scan (MUGA), no abnormal findings requiring treatment on electrocardiogram within 21 days before enrollment, ability to ingest orally, life expectancy of greater than 3 months, and provision of written informed consent. Exclusion criteria were as follows: contraindication for S-1, cisplatin, or trastuzumab, pregnant or possibly pregnant women, men who wanted their partners to become pregnant, patients with active infection, patients with active hepatitis type B infection, serious illness or medical conditions with a previous history of congestive heart failure, hi-risk uncontrolled arrhythmias, unstable angina requiring medication, a previous history of myocardial infarction, severe heart valve disease, a previous history of transmural infarct, or uncontrolled hypertension, serious complication with interstitial pneumonia, pulmonary fibrosis, heart failure, renal failure, hepatic failure, or uncontrolled diabetes mellitus, patients with resting dyspnea, patients with new hemorrhage from gastric cancer and/or the digestive tract, patients with diarrhea (four or more times per day or watery stool), second primary malignancy (except those adequately treated basal cell carcinoma treated more than five years ago without recurrence), patients receiving continuous systemic administration of flucytosine, phenytoin or warfarin, patients receiving systemic administration of corticosteroid, or any patients judged by the investigator to be unfit to participate in the study.

The study protocol was approved by the Osaka Gastrointestinal Cancer Chemotherapy Study Group (OGSG) Steering Committee (OGSG1202) and the institutional review boards of all participating institutions. All patients provided written informed consent before enrollment. This study was registered with UMINCTR, UMIN000007941.

Treatment. Patients received cisplatin $\left(60 \mathrm{mg} / \mathrm{m}^{2}\right)$ plus trastuzumab (course 1,8 mg/kg; course 2 onward, $6 \mathrm{mg} / \mathrm{kg}$ ) intravenously on day 1 and oral S-1 twice daily at a dose based on the body surface area $\left(<1.25 \mathrm{~m}^{2}, 40 \mathrm{mg} ; \geq 1.25\right.$ to $\left.<1.5 \mathrm{~m}^{2}, 50 \mathrm{mg} ; \geq 1.5 \mathrm{~m}^{2}, 60 \mathrm{mg}\right)$ on days 1-14 of a 21-day cycle. This schedule was repeated until disease progression, development of unacceptable toxicity, or patient withdrawal of consent. If patients had a neutrophil count $<1,000 / \mathrm{mm}^{3}$, platelet count $<75 \times 10^{3} / \mathrm{mm}^{3}$, serum creatinine $>1.2 \mathrm{mg} / \mathrm{dl}$, infection with fever, or anorexia, diarrhea, oral mucositis, or rash of grade 2 or higher, treatment with S-1 was suspended. For patients with febrile neutropenia, grade 4 neutropenia, grade 3-4 thrombocytopenia, serum creatinine $>1.2 \mathrm{mg} / \mathrm{dl}$, or grade 3-4 diarrhea, oral mucositis, or rash, doses of S-1 and cisplatin were reduced starting from the next cycle. For patients who had grade 3-4 vomiting or anorexia because of cisplatin, its dose was reduced. If heart failure or severe infusion reactions occurred, trastuzumab was discontinued.

End points and assessments. The primary end point of the study was OS, with secondary end points including adverse events, progression-free survival (PFS), and time to treatment failure (TTF). OS was defined as the time from the date of enrollment to the date of death from any cause. PFS was defined as the time from the date of enrollment to the date of disease progression or death from any cause. TTF was defined as the time from the date of enrollment to the date when the treating physician decided to discontinue treatment for any reason. Physical examination and blood tests were mandatory before each course, and left ventricular ejection fraction was assessed every 3 months during treatment. Adverse events were evaluated according to the National Cancer Institute Common Terminology Criteria for Adverse Events, version 4.0.

Statistical analysis. The required sample size was estimated based on a threshold median survival time (MST) of 13 months and an expected MST of 19 months, $80 \%$ power, and an alpha value of 0.1 (one-sided) using the binomial test. Considering ineligible patients to comprise $2 \%$, the target sample size was determined to be at least 40 patients.

We used the Kaplan-Meier method to estimate survival curves and Greenwood's formula to calculate the $95 \%$ confidence interval (CI) 
for survival rates. Statistical analyses were conducted with R, version 3.5.1. (The R Foundation for Statistical Computing, Vienna, Austria).

\section{Results}

Patients. Enrollment was started in February 2013, and terminated after the inclusion of 15 patients from 12 institutions in May 2016 as a result of slow accrual. One patient committed protocol deviation with an inadequate neutrophil count of $1,800 / \mathrm{mm}^{3}$ before protocol treatment. Including this case, the characteristics of the 15 patients are listed in Table I. They included 12 males and 3 females. The median age was 66 years (range $=51-75$ years). Eight patients had a past history of gastrectomy. Twelve patients had unresectable lesions, and three patients had recurrent disease. Ten patients had IHC $3+$ tumors, and five patients had IHC $2+/$ FISH-positive tumors.

Efficacy. Analysis was conducted two years after closure of recruitment, at which time (May 2018) all patients had discontinued treatment. The median number of cycles was 7 (range $=1-51$ cycles), and the median relative dose intensity for $\mathrm{S}-1$, cisplatin, and trastuzumab was $72 \%, 76 \%$, and $94 \%$, respectively. The main reason for treatment discontinuation was progressive disease (eight patients, 53\%), followed by adverse events (six patients, 40\%), and patient refusal due to adverse events (one patient).

At the time of analysis, 12 patients had died. The median duration of follow-up for the three surviving patients was 44.9 months. The median OS was 14.4 months $(95 \% \mathrm{CI}=10.3$ months-not applicable, one-sided $90 \% \mathrm{CI}=12.3$ months (Figure 1). The 1- and 3-year OS rates were $66.7 \%$ $(95 \% \mathrm{CI}=46.6-95.3 \%)$ and $26.7 \%(95 \% \mathrm{CI}=11.5-61.7 \%)$, respectively. The median PFS was 9.8 months $(95 \% \mathrm{CI}=8.6-$ 36.6 months; Figure 2). The 1 and 3 -year PFS rates were $33.3 \%(95 \% \mathrm{CI}=16.3-68.2 \%)$ and $20.0 \%(95 \% \mathrm{CI}=7.3-$ $55.0 \%)$, respectively. The median TTF was 6.1 months (95\% CI=3.0-12.5 months; Figure 3). The 6-month and 1year TTF rates were $46.7 \%(95 \% \mathrm{CI}=27.2-80.2 \%)$ and $20.0 \%$ $(95 \% \mathrm{CI}=7.3-55.0 \%)$, respectively.

Safety. There were no deaths during the protocol or within 30 days after the protocol treatment. Hematological and nonhematological adverse events are listed in Table II. The most common hematological adverse events were leukopenia (any grade, 93\%; grade 3-4, 20\%), neutropenia (any grade, 93\%; grade $3-4,47 \%$ ), and anemia (any grade, $80 \%$; grade $3-4$, $40 \%$ ). No other grade 3-4 hematological adverse events were observed. Regarding non-hematological toxicities, fatigue $(87 \%)$ and anorexia $(87 \%)$ were the most common all-grade adverse events. Diarrhea (20\%), nausea (20\%), and anorexia (20\%) were the most common grade 3-4 events. Heart failure did not occur.
Table I. Eligible patient characteristics

\begin{tabular}{lc}
\hline Characteristics & $\mathrm{n}=15$ \\
\hline Age, years & \\
Median & 66 \\
Range & $51-75$ \\
Gender & \\
Male & $12(80 \%)$ \\
Female & $3(20 \%)$ \\
Performance status & \\
0 & $7(47 \%)$ \\
1 & $8(53 \%)$ \\
Previous gastrectomy & $7(47 \%)$ \\
No & $1(7 \%)$ \\
Total gastrectomy & $7(47 \%)$ \\
Distal gastrectomy & \\
Unresectable/recurrent & $12(80 \%)$ \\
Unresectable & $2(13 \%)$ \\
Recurrent with adjuvant chemotherapy & $1(7 \%)$ \\
Recurrent without adjuvant chemotherapy & \\
Histological type & $8(53 \%)$ \\
Differentiated & $7(47 \%)$ \\
Undifferentiated & $10(67 \%)$ \\
HER2 status & $5(33 \%)$ \\
IHC 3+ & \\
IHC 2+/FISH positive & $15(100 \%)$ \\
Tumor location & 0 \\
Stomach & \\
Esophagogastric junction & \\
\hline
\end{tabular}

\section{Discussion}

In this study, 40 patients were initially planned to be enrolled within two years. However, because of slow accrual, recruiting was terminated with 15 patients at three years and three months. The MST was 14.4 months and the lower limit of the one-sided $90 \%$ CI was 12.3 months, which was slightly shorter than the preplanned null hypothesis threshold of 13 months.

Although the accrual of patients was insufficient in this study, its efficacy was acceptable considering the 1-year survival rate of $66.7 \%$, the median PFS of 9.8 months, and the median TTF of 6.1 months, compared with the HERBIS1 trial (MST, 16.0 months; 1-year survival rate, 67.9\%; median PFS, 7.8 months; median TTF, 5.7 months) (5) and the ToGA trial Japanese subgroup that received trastuzumab with capecitabine plus cisplatin (MST, 15.9 months; 1-year survival rate, 68\%; median PFS, 6.2 months) (17).

There were some differences in patient characteristics between the current HERBIS-1B trial and the HERBIS-1 trial. More patients with PS 1 participated in the HERBIS$1 \mathrm{~B}$ trial $(53 \%)$ than in the HERBIS-1 trial $(22 \%)$, more patients with undifferentiated-type cancer participated in the HERBIS-1B trial (47\%) than in the HERBIS-1 trial (33\%), and fewer patients with IHC $3+$ tumors participated in the 


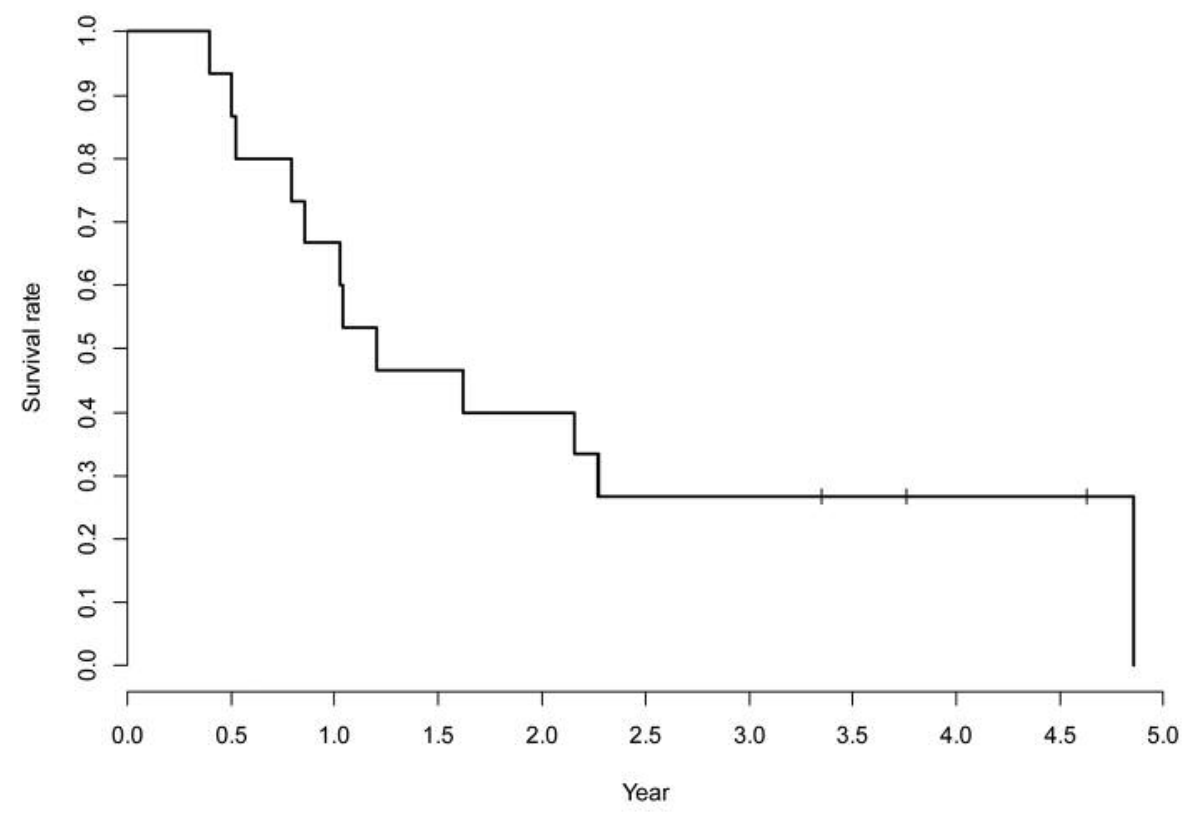

Figure 1. The Kaplan-Meier overall survival curve.

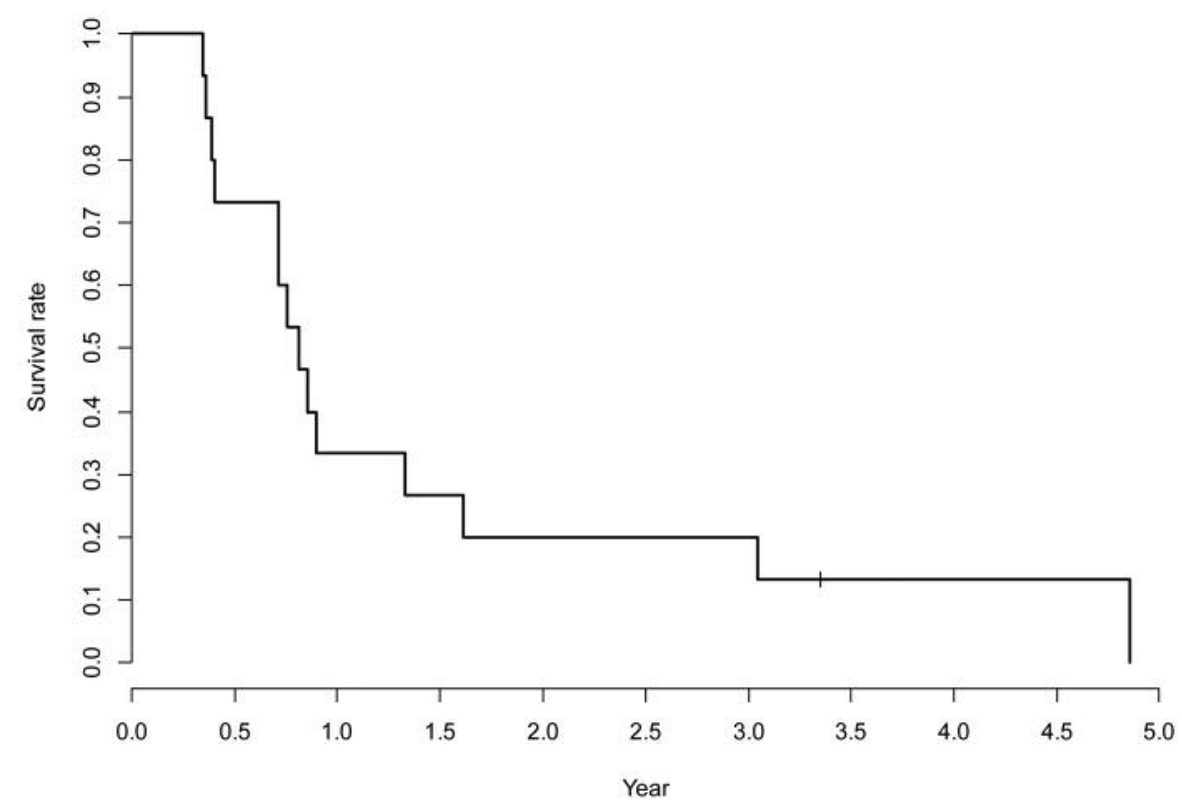

Figure 2. The Kaplan-Meier progression-free survival curve.

HERBIS-1B trial (67\%) than in the HERBIS-1 trial (83\%). Undifferentiated-type cancer is considered to be associated with a poorer prognosis than differentiated-type cancer (17). Patients with IHC $3+$ tumors were more likely to survive longer than those with IHC 2+/FISH-positive tumors when treated with trastuzumab (3). These factors may have influenced the OS in the current study.
The toxicity profiles in the current study were comparable with those in the HERBIS-1 trial and the ToGA trial Japanese subgroup; however, there were some differences in the frequency and severity of adverse events. Grade 3-4 anemia was more common in the HERBIS-1B trial (40\%) than in the HERBIS-1 trial (15\%) and the ToGA trial Japanese subgroup (25\%). Grade 3-4 nausea was more 


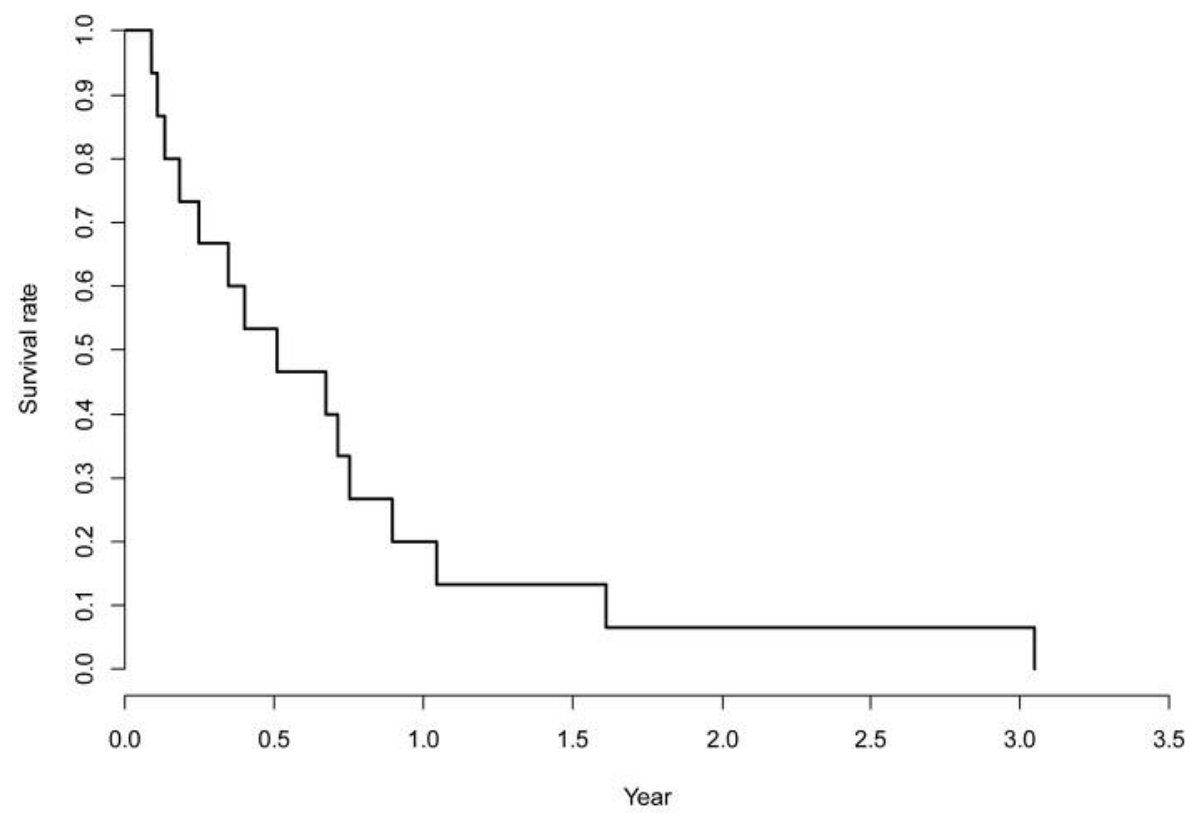

Figure 3. The Kaplan-Meier time to treatment failure curve.

common in the HERBIS-1B trial $(20 \%)$ than in the HERBIS1 trial $(2 \%)$ and the ToGA trial Japanese subgroup (14\%). Grade 3-4 diarrhea was also more common in the HERBIS1B trial $(20 \%)$ than in the HERBIS-1 trial $(8 \%)$ and the ToGA trial Japanese subgroup $(8 \%)$. These differences in adverse events must be interpreted with caution because of the differences among the groups in terms of patient characteristics. In particular, previous gastrectomy was significantly more prevalent in the HERBIS-1B trial (53\%) than in the HERBIS- 1 trial $(17 \%)$ and the ToGA trial Japanese subgroup (16\%). A past history of gastrectomy may have affected these observed adverse events.

Since the ToGA trial, several phase II studies have been carried out to evaluate the efficacy of different chemotherapeutic agents in combination with trastuzumab for HER-2-positive AGC; i.e., SP (5, 7-9), capecitabine plus oxaliplatin $(10,11)$, docetaxel plus cisplatin plus S-1(12), S-1 (13), docetaxel plus S-1 (14), docetaxel plus oxaliplatin plus fluorouracil (15), and docetaxel plus cisplatin plus fluorouracil (16). Chua et al. (7) investigated the efficacy of tri-weekly SP plus trastuzumab, and reported a MST of 14.6 months. Kataoka et al. (8) and the WJOG7212G trial (9) administered five-weekly SP plus trastuzumab, and reported a MST of 15.3 and 16.5 months, respectively. Kataoka et al. included patients without measurable lesions, but they accounted for only 3 out of 30. The other studies enrolled patients with measurable lesions only, except one which included a patient without measurable lesions (12). Thus, the current study was the first phase II trial to evaluate the efficacy and safety of
Table II. Adverse events

\begin{tabular}{|c|c|c|c|c|c|c|}
\hline \multirow[t]{2}{*}{ Event } & \multicolumn{6}{|c|}{ Grade } \\
\hline & 1 & 2 & 3 & 4 & Any $(\%)$ & $3-4(\%)$ \\
\hline Leukopenia & 4 & 7 & 3 & 0 & 93 & 20 \\
\hline Neutropenia & 1 & 6 & 6 & 1 & 93 & 47 \\
\hline Anemia & 3 & 3 & 6 & 0 & 80 & 40 \\
\hline Thrombocytopenia & 6 & 2 & 0 & 0 & 53 & 0 \\
\hline Creatinine increased & 6 & 4 & 0 & 0 & 67 & 0 \\
\hline Blood bilirubin increased & 1 & 0 & 0 & 0 & 7 & 0 \\
\hline AST increased & 6 & 0 & 0 & 0 & 40 & 0 \\
\hline ALT increased & 4 & 1 & 0 & 0 & 33 & 0 \\
\hline Hypoalbuminemia & 3 & 7 & 0 & 0 & 67 & 0 \\
\hline Hyponatremia & 1 & 0 & 0 & 0 & 7 & 0 \\
\hline Diarrhea & 4 & 2 & 3 & 0 & 60 & 20 \\
\hline Oral mucositis & 5 & 1 & 0 & 0 & 40 & 0 \\
\hline Skin rash & 2 & 1 & 0 & 0 & 20 & 0 \\
\hline Nausea & 4 & 1 & 3 & 0 & 53 & 20 \\
\hline Vomiting & 1 & 1 & 1 & 0 & 20 & 7 \\
\hline Fatigue & 7 & 4 & 2 & 0 & 87 & 13 \\
\hline Anorexia & 6 & 4 & 3 & 0 & 87 & 20 \\
\hline Conjunctivitis & 0 & 1 & 0 & 0 & 7 & 0 \\
\hline Interstitial pneumonia & 0 & 0 & 1 & 0 & 7 & 7 \\
\hline Hypertension & 0 & 0 & 1 & 0 & 7 & 7 \\
\hline Thromboembolic event & 0 & 0 & 1 & 0 & 7 & 7 \\
\hline Hand-foot syndrome & 0 & 2 & 0 & 0 & 13 & 0 \\
\hline Fever & 0 & 1 & 0 & 0 & 7 & 0 \\
\hline Nasal discharge & 1 & 0 & 0 & 0 & 7 & 0 \\
\hline Abdominal abscess & 0 & 1 & 0 & 0 & 7 & 0 \\
\hline Constipation & 1 & 1 & 0 & 0 & 13 & 0 \\
\hline Peripheral sensory neuropathy & 0 & 2 & 0 & 0 & 13 & 0 \\
\hline
\end{tabular}


chemotherapy in combination with trastuzumab for HER-2positive AGC without measurable lesions.

The limitation of this study was the insufficient enrollment of patients. HER2-positive AGC without measurable lesions was regarded as a minor population, as demonstrated in the ToGA trial in which only $9.8 \%$ of patients did not have measurable lesions. Undifferentiated-type gastric cancer, comprising $47 \%$ of our enrolled patients, is less likely to be associated with HER2 alteration (3.1\%) than differentiated-type cancer (18.1\%) (18). Therefore, further investigation with prospective clinical trials may be difficult for this small population with HER2-positive AGC without measurable lesions.

In conclusion, trastuzumab with S-1 and cisplatin may be effective and tolerable for patients with HER2-positive advanced gastric cancer with or without measurable lesions.

\section{Conflicts of Interest}

S. Endo has received a speaker honorarium from Chugai Pharmaceutical Co., Ltd., Bristol-Myers Squibb K.K., and Eli Lilly Japan K.K. Y. Kurokawa has received a speaker honorarium from Taiho Pharmaceutical Co., Ltd. and Chugai Pharmaceutical Co., Ltd. D. Sakai has received research grants from Chugai Pharmaceutical Co., Ltd., ONO Pharmaceutical Co., Ltd., Eli Lilly, and Daiichi Sankyo; a speaker honorarium from Chugai Pharmaceutical Co., Ltd., and departmental research grants from Chugai Pharmaceutical Co., Ltd., ONO Pharmaceutical Co., Ltd., and Yakult Honsha Co., Ltd.. T. Satoh has received consulting fees from Eli Lilly and Daiichi Sankyo and consulting fees and honoraria from Chugai Pharmaceutical Co., Ltd., Merck Serono Co., Ltd., Bristol-Myers K.K., Taiho pharmaceutical Co., Ltd., and Takeda Pharmaceutical Co., Ltd., and departmental research grants from Chugai Pharmaceutical Co., Ltd., ONO Pharmaceutical. Co., Ltd., and Yakult Honsha Co., Ltd.. The other authors indicated no financial relationships.

\section{Authors' Contributions}

Y. Kurokawa wrote the protocol. T. Satoh, Y. Kurokawa, and D. Sakai chaired the study group. S. Endo, Y. Kurokawa, M. Gamoh, Y. Kimura, J. Matsuyama, H. Taniguchi, A. Takeno, R. Kawabata, J. Kawada, T. Masuzawa, K. Yamamoto, and K. Kobayashi gathered the data. T. Shimokawa analyzed the data. All Authors were involved in the drafting, review, and approval of the manuscript and the decision to submit for publication.

\section{Acknowledgements}

This study was conducted by the Osaka Gastrointestinal Cancer Chemotherapy Study Group (OGSG).

\section{References}

1 World Health Organization: Cancer. http://www.who.int/newsroom/fact-sheets/detail/cancer Accessed 4 Dec, 2018.

2 Cancer Information Service, National Cancer Center, Japan: Cancer Registry and Statistics. https://ganjoho.jp/reg_stat/ statistics/stat/summary.html Accessed 4 Dec, 2018.
3 Bang YJ, Van Cutsem E, Feyereislova A, Chung HC, Shen L, Sawaki A, Lordick F, Ohtsu A, Omuro Y, Satoh T, Aprile G, Kulikov E, Hill J, Lehle M, Rüschoff $J$ and Kang YK: Trastuzumab in com-bination with chemotherapy versus chemotherapy alone for treatment of HER2-positive advanced gastric or gastro-oesophageal junction cancer (ToGA): a phase 3, open-label, randomised controlled trial. Lancet 376: 687697, 2010. PMID: 20728210, DOI: 10.1016/S01406736(10)61121-X

4 Koizumi W, Narahara H, Hara T, Takagane A, Akiya T, Takagi M, Miyashita K, Nishizaki T, Kobayashi O, Takiyama W, Toh Y, Nagaie T, Takagi S, Yamamura Y, Yanaoka K, Orita H and Takeuchi M: S-1 plus cisplatin versus S-1 alone for first-line treatment of advanced gastric cancer (SPIRITS trial): a phase III trial. Lancet Oncol 9: 215-221, 2008. PMID: 18282805, DOI: 10.1016/S1470-2045(08)70035-4

5 Kurokawa Y, Sugimoto N, Miwa H, Tsuda M, Nishina S, Okuda H, Imamura H, Gamoh M, Sakai D, Shimokawa T, Komatsu Y, Doki Y, Tsujinaka $\mathrm{T}$ and Furukawa H: Phase II study of trastuzumab in combination with S-1 plus cisplatin in HER2positive gastric cancer (HERBIS-1). Br J Cancer 110: 11631168, 2014. PMID: 24473399, DOI: $10.1038 /$ bjc 2014.18

6 Eisenhauer EA, Therasse P, Bogaerts J, Schwartz LH, Sargent D, Ford R, Dancey J, Arbuck S, Gwyther S, Mooney M, Rubinstein L, Shankar L, Dodd L, Kaplan R, Lacombe D and Verweij J: New response evaluation criteria in solid tumours: revised RECIST guideline (version 1.1). Eur J Cancer 45: 228-247, 2009. PMID: 19097774, DOI: 10.1016/j.ejca. 2008.10.026

7 Chua C, Tan IB, Yamada Y, Rha SY, Yong WP, Ong WS, Tham CK, Ng M, Tai DW, Iwasa S, Lim HY and Choo SP: Phase II study of trastuzumab in combination with S-1 and cisplatin in the first-line treatment of human epidermal growth factor receptor HER2-positive advanced gastric cancer. Cancer Chemother Pharmacol 76: 397-408, 2015. PMID: 26099969, DOI: $10.1007 / \mathrm{s} 00280-015-2811-y$

8 Kataoka H, Mori Y, Shimura T, Nishie H, Natsume M, Mochizuki H, Hirata Y, Sobue S, Mizushima T, Sano H, Mizuno Y, Nakamura M, Hirano A, Tsuchida K, Adachi K, Seno K, Kitagawa M, Kawai T and Joh T: A phase II prospective study of the trastuzumab combined with 5-weekly S-1 and CDDP therapy for HER2-positive advanced gastric cancer. Cancer Chemother Pharmacol 77: 957-962, 2016. PMID: 27002325, DOI: 10.1007/s00280-016-3013-y

9 Miura Y, Sukawa Y, Hironaka S, Mori M, Nishikawa K, Tokunaga S, Okuda H, Sakamoto T, Taku K, Nishikawa K, Moriwaki T, Negoro Y, Kimura Y, Uchino K, Shinozaki K, Shinozaki H, Musha N, Yoshiyama H, Tsuda T, Miyata Y, Sugimoto N, Shirakawa T, Ito M, Yonesaka K, Yoshimura K, Boku N, Nosho K, Takano T and Hyodo I: Five-weekly S-1 plus cisplatin therapy combined with trastuzumab therapy in HER2positive gastric cancer: a phase II trial and biomarker study (WJOG7212G). Gastric Cancer 21: 84-95, 2018. PMID: 28497176, DOI: $10.1007 / \mathrm{s} 10120-017-0725-6$

10 Ryu MH, Yoo C, Kim JG, Ryoo BY, Park YS, Park SR, Han HS, Chung IJ, Song EK, Lee KH, Kang SY and Kang YK: Multicenter phase II study of trastuzumab in combination with capecitabine and oxaliplatin for advanced gastric cancer. Eur $\mathrm{J}$ Cancer 51: 482-488, 2015. PMID: 25661103, DOI: 10.1016/j.ejca.2014.12.015 
11 Gong J, Liu T, Fan Q, Bai L, Bi F, Qin S, Wang J, Xu N, Cheng Y, Bai Y, Liu W, Wang L and Shen L: Optimal regimen of trastuzumab in combination with oxaliplatin/ capecitabine in first-line treatment of HER2-positive advanced gastric cancer (CGOG1001): a multicenter, phase II trial. BMC Cancer 16: 68, 2016. PMID: 26857702, DOI: 10.1186/s12885-016-2092-9

12 Mitsui Y, Sato Y, Miyamoto H, Fujino Y, Takaoka T, Miyoshi J, Kagawa M, Ohnuma H, Hirakawa M, Kubo T, Osuga T, Sagawa T, Sato Y, Takahashi Y, Katsuki S, Okuda T, Takimoto R, Kobune M, Nobuoka T, Hirata K, Kato J and Takayama T: Trastuzumab in combination with docetaxel/cisplatin/S-1 (DCS) for patients with HER2-positive metastatic gastric cancer: feasibility and preliminary efficacy. Cancer Chemother Pharmacol 76: 375-382, 2015. PMID: 26099968, DOI: 10.1007/s00280-015-2807-7

13 Kimura Y, Fujii M, Masuishi T, Nishikawa K, Kunisaki C, Matsusaka S, Segawa Y, Nakamura M, Sasaki K, Nagao N, Hatachi Y, Yuasa Y, Asami S, Takeuchi M, Furukawa H and Nakajima T: Multicenter phase II study of trastuzumab plus S-1 alone in elderly patients with HER2-positive advanced gastric cancer (JACCRO GC-06). Gastric Cancer 21: 421-427, 2018. PMID: 28936560, DOI: 10.1007/s10120-017-0766-X

14 Kagawa S, Muraoka A, Kambara T, Nakayama H, Hamano R, Tanaka N, Noma K, Tanakaya K, Kishimoto H, Shigeyasu K, Kuroda S, Kikuchi S, Kuwada K, Nishizaki M, Shirakawa Y and Fujiwara T: A multi-institution phase II study of docetaxel and S-1 in combination with trastuzumab for HER2-positive advanced gastric cancer (DASH study). Cancer Chemother Pharmacol 81: 387-392, 2018. PMID: 29290024, DOI: 10.1007/s00280-017-3505-4

15 Roviello G, Petrioli R, Nardone V, Rosellini P, Multari AG, Conca $\mathrm{R}$ and Aieta M: Docetaxel, oxaliplatin, 5FU, and trastuzumab as first-line therapy in patients with human epidermal receptor 2-positive advanced gastric or gastroesophageal junction cancer: Preliminary results of a phase II study. Medicine (Baltimore) 97: e10745, 2018. PMID: 29768350, DOI: 10.1097/MD.0000000000010745
16 Mondaca S, Margolis M, Sanchez-Vega F, Jonsson P, Riches JC, Ku GY, Hechtman JF, Tuvy Y, Berger MF, Shah MA, Kelsen DP, Ilson DH, Yu K, Goldberg Z, Epstein AS, Desai A, Chung V, Chou JF, Capanu M, Solit DB, Schultz N and Janjigian YY: Phase II study of trastuzumab with modified docetaxel, cisplatin, and 5 fluorouracil in metastatic HER2-positive gastric cancer. Gastric Cancer, 2018. [Epub ahead of print] PMID: 30088161, DOI: $10.1007 / \mathrm{s} 10120-018-0861-7$

17 Sawaki A, Ohashi Y, Omuro Y, Satoh T, Hamamoto Y, Boku N, Miyata Y, Takiuchi H, Yamaguchi K, Sasaki Y, Nishina T, Satoh A, Baba E, Tamura T, Abe T, Hatake K and Ohtsu A: Efficacy of trastuzumab in Japanese patients with HER2-positive advanced gastric or gastroesophageal junction cancer: a subgroup analysis of the Trastuzumab for Gastric Cancer (ToGA) study. Gastric Cancer 15: 313-322, 2012. PMID: 22179434, DOI: 10.1007/s10120-011-0118-1

18 Oh HS, Eom DW, Kang GH, Ahn YC, Lee SJ, Kim JH, Jang HJ, Kim EJ, Oh KH and Ahn HJ: Prognostic implications of EGFR and HER-2 alteration assessed by immunohistochemistry and silver in situ hybridization in gastric cancer patients following curative resection. Gastric Cancer 17: 402-411, 2014. PMID: 23955257, DOI: $10.1007 / \mathrm{s} 10120-013-0288-0$

Received December 28, 2018

Revised January 14, 2019

Accepted January 15, 2019 